\title{
RAPPORT DE LA SESSION MIXTE 30 ET 42
}

\author{
Monday, 28 August 1967 - 8:45 a.m. to 10:15 a.m.
}

CharRman: A.H. Batten.

SECRETARY: J. M. Fletcher.

Ninety-six persons attended this session which was devoted to a discussion of the 'Determination of Orbital Elements and Stellar Masses'.

The Chairman introduced the session by explaining that the 30-42 Joint Working Group had felt that the time was ripe for a discussion of how far the derived orbital elements of close binary systems represented the true physical nature of the systems. Not all the speakers invited had agreed that this was an important topic, but he hoped this topic might emerge in discussion. He discussed the distribution of the longitudes of periastron in close binaries, as found in the recently completed Sixth Catalogue of the Orbital Elements of Spectroscopic Binary Systems. This distribution clearly shows non-random features in addition to the well-known Barr Effect, and clearly indicates that the longitude of periastron is often a mathematical parameter of the velocitycurve rather than a true measure of the orientation of the orbit. He briefly described the new Catalogue.

D. Ya. Martynov expressed his concern that many observatories in the United States had apparently not received copies of his Bibliography of Spectroscopic Binaries and asked all participants to check this matter on their return home and to notify him if they had not received their copies. He discussed the work of Dr Cherepaschuk on the problem of eclipsing binaries with extended atmospheres, with particular reference to the Wolf-Rayet system V444 Cygni. It had been possible to obtain consistent solutions from four-colour photoelectric observations. The electron density in the atmosphere appeared to follow an exponential relation not in agreement with that derived by Chandrasekhar.

$R$. H. Koch reported on the co-operative programme of observations of SX Cassiopeiae sponsored by Commission 42 during 1964-67. Narrow-band and UBV photometry have shown the period of the system to have been constant since its discovery. The light-curve is intrinsically variable, but the width of primary eclipse, at a given time, is independent of the wavelength of observation. Analysis of the light-curve will probably prove to be very difficult. Over one hundred old spectrograms obtained by Joy have been discovered in the Mount Wilson Observatory files. The velocity curves compiled from these do not agree with Struve's published results. The star has a faint companion of possibly variable radial velocity. Astrometric observations, however, indicate that this companion is optical.

K.O. Wright discussed recent Victoria observations of 32 Cygni. Both photoelectric and spectroscopic observations are desired during the interval August 1 to November 1, 1968 to cover the eclipse of September 1968. Variations in the satellites of the K-line have been found, and it is desirable to establish the time-scale of these. The chromospheric lines in the spectra of 31 Cygni, 32 Cygni and VV Cephei seem to show large changes of intensity from eclipse to eclipse. It is not clear whether these are real changes or of instrumental origin.

$M$. Plavec pleaded for more observations of bright eclipsing binaries. The theory of close binary systems has made great strides in the last few years and the need for new and better observational data is urgent. Of 191 systems brighter than $8^{m} .5$ at maximum light, it is impossible to say of 46 whether they are Algol-type or main-sequence systems. For a further 59 the available information permits only a tentative classification. He intended to circulate a list of systems for which observations are particularly desirable. Martynov questioned the value of such lists, in his experience they had produced little or no response. Plavec felt that a short list, with notes on each system might serve to stimulate interest among observers. The question of the availability of suitable comparison stars 
for bright systems was raised. Plavec was of the opinion that this would be no problem in the vast majority of cases.

D.M. Popper emphasized the importance of using sufficiently high spectrographic dispersions for the determination of stellar masses. A dispersion of at least $20 \AA / \mathrm{mm}$, and often of $10 \AA / \mathrm{mm}$, is necessary for this purpose. He reiterated Plavec's remarks on the need for new photometric observations. He felt that such observations should be on a well-standardized colour system-such as $U B V$ - and usually in at least two colours. He commented on the existence of an apparent group of systems in which both components are subgiants of spectral types $G$ or early $K$ and which show $\mathrm{H}$ and $\mathrm{K}$ emission in one or both spectra. The system AR Lacertae is the best known example. In some of these systems, SS Bootis being a case in point, both evolved components may be undermassive for their luminosity with masses less than one solar mass. The so-called R Canis Majoris systems are binaries with both components reportedly having abnormally low masses for their main-sequence spectral types. For one of these systems, TU Monocerotis, the K-line of the secondary spectrum blends with an interstellar line on plates of inadequate dispersion. Use of adequate dispersion removes the mass anomaly. Koch commented that it is difficult to standardize one's photometric system each night. Popper replied this was not necessary so long as the observations were on a standard system.

$M . W$. Ovenden presented a discussion by himself and A.H. Batten on the mass data in the Sixth Catalogue. Masses $(m)$ have been computed for those systems in the Catalogue for which inclinations can be found from visual or eclipsing orbits, and for which absolute magnitudes can be found from visual orbits, trigonometric parallaxes or $\mathrm{H} \gamma$ equivalent widths. For absolute visual magnitudes $-6<M_{v}<+2$, the $\left(M_{v}, \log m\right)$ relation is linear, with no systematic differences between the magnitudes derived from $\mathrm{H} \gamma$ and those derived from dynamical and trigonometric parallaxes. For $M_{v}>+4$ there is slight evidence for a dual $\left(M_{v}, \log m\right)$ relation. The observed $\left(M_{\mathrm{bol}}, \log m\right)$ relation is in good agreement with a series of main-sequence models with $(\mathrm{X}, \mathrm{Y})=$ $(0 \cdot 68,0 \cdot 30)$.

Mrs A.E. Ringuelet-Kaswalder presented work by herself and J. Sahade on the determination of masses of Algol-type systems. Two methods are commonly used. Either one assumes that the primary component is a main-sequence star, or one assumes that the secondary component fills its equipotential lobe. In the second method, the mass-ratio derived is very sensitive to the assumed radius of the lobe, and therefore quite small photometric errors in the determination of the radius of the secondary component can lead to serious errors in the derived masses. Uniform use of the first method might eliminate the apparent anomaly of the $\mathbf{R}$ Canis Majoris systems. Plavec commented that the important point was to obtain good observations. For 19 out of 25 systems with well-determined elements, the two possible assumptions led to closely similar masses. Sahade replied that such agreement was to be expected in many cases, but the second method could fail badly; and, furthermore, it should be remembered that the equipotential surfaces refer to ideal stars and not to real ones.

A later communication was received from V.P. Cesevič concerning recent work on eclipsing binaries in the U.S.S.R. V. Tabačnik (Odessa) has worked on the computation of elements by means of an electronic computer, and has applied his method to S Cancri. M. Kiperman and A. Sulberg (Odessa) have investigated the possibility of determining the non-linear coefficients of limb darkening, as discussed by Grygar. Cesevič himself has investigated the analysis of atmospheric eclipses with particular reference to the systems RW Trianguli and U Geminorum. He found much difficulty in the use of Linnell's tables and questions whether they can represent an observed light-curve at all. Poostylnik (Tartu) has also considered the problems of atmospheric eclipses, and computed tables based on theoretical values of the coefficient of absorption. 